\title{
Optical Tweezers manipulation of colloids and biopolymers: non-equilibrium processes
}

\author{
G.M. Wang and E.M. Sevick \\ Research School of Chemistry, The Australian National University, Canberra 0200 ACT, \\ Australia
}

\begin{abstract}
The Fluctuation Theorems (FTs) of Evans \& Searles and of Crooks are fundamental theorems of modern thermodynamics that have been suggested to be of practical use to scientists and engineers. Non-equilibrium processes with energy fluctuations on the order of thermal energy, $k_{B} T$, are described by the FTs; examples include the stretching of a DNA molecule, the localisation of a colloidal particle in an optical trap of changing strength, and translation of an optically trapped colloidal particle. If the path or process is traversed over long times or the system is sufficiently large that it can be considered in the classical, thermodynamic limit, then, in principle, there is only one value of the energy characterising the path. However, for small systems, there exists a distribution of energy values and this distribution is associated with non-equilibrium fluctuations of the system that do not average out over short time. The FT of Evans \& Searles, as well as the FT of Crooks (from which the Jarzynski relation is derived), describe the symmetry of this energy distribution about zero. This distribution is inherent to the dynamics of small systems, such as nano-machines and single molecular motors.

In this paper we present the FTs in a single unified language, considering that the work done on the system is either purely dissipative, achieves a change in thermodynamic state of the system, or a combination of these. We demonstrate this with a single colloidal particle in an optical trap and a single DNA molecule stretched in an OT experiment.
\end{abstract}

Keywords: Fluctuation Theorems, single molecule DNA stretching, Optical Tweezers

\section{INTRODUCTION}

Thermodynamics is the study of the flow of heat and the transformation of work into heat. Our understanding of thermodynamics is largely confined to equilibrium states. Linear irreversible thermodynamics is an extension of the 19th century concepts of equilibrium thermodynamics to systems that are sufficiently close to equilibrium that intensive thermodynamic variables can be approximated by the same functions of local state variables, as would be the case if the entire system was in complete thermodynamic equilibrium. ${ }^{1,2}$ Moreover these traditional concepts are limited in application to large systems or averages over an ensemble of states, referred to as the "thermodynamic limit". Inventors and engineers endeavour to scale down machines and devices to nanometer sizes for a wide range of technological purposes. However, there is a fundamental limit to miniaturisation since small engines are not simply re-scaled versions of their larger counterparts. If the work performed during the duty cycle of any machine is comparable to thermal energy per degree of freedom, then one can expect that the machine will operate in "reverse" over short time scales. That is, heat energy from the surroundings will be converted into useful work allowing the engine to run backwards. For larger engines, we would describe this as a violation of the Second Law of thermodynamics, as entropy is consumed rather than generated. Until recently, this received little attention in the nanotechnology literature, as there was no theory capable of describing the probability of entropy consumption in such small engines.

In the last fifteen years, several fluctuation theorems have been proposed that revolutionise our understanding and use of thermodynamics. Firstly these new theorems lift the requirement of the thermodynamic limit. This allows thermodynamic concepts to be applied to finite, even small systems. Secondly, these new theorems can be

Further author information:

EMS: E-mail: sevick@rsc.anu.edu.au.

GMW: E-mail: gmw@rsc.anu.edu.au 
applied to systems that are arbitrarily far from equilibrium. Thirdly, these theorems explain how macroscopic irreversibility appears naturally in systems that obey time reversible microscopic dynamics: such a resolution of the Loschmidt (Irreversibility) Paradox had defied our best efforts for more than 100 years. One of these fluctuation theorems, the Evans-Searles Fluctuation Theorem (Evans-Searles FT), ${ }^{3,4}$ results in a generalisation of the Second Law of Thermodynamics so that it applies to small systems, including those that evolve far from equilibrium. Another, the Crooks Fluctuation Theorem (Crooks FT) ${ }^{5,6}$ provides a method of predicting equilibrium free energy difference from experimental information taken from nonequilibrium paths that connect two equilibrium states. This FT can be used to derive the well known Jarzynski Equality, ${ }^{7}$ which expresses the free energy difference between two equilibrium states in terms of an average over irreversible paths. Both FTs are at odds with a traditional understanding of 19th century thermodynamics. Nevertheless, these theorems will be essential for the application of thermodynamic concepts to nanotechnology systems which are currently of such interest to biologists, physical scientists and engineers.

In 2002 the FTs were demonstrated experimentally by two independent groups, each with a unique focus and both using optical tweezers. Wang et $a l^{9}$ demonstrated the Evans-Searles FT by monitoring the transient trajectory of a single colloidal bead in a translating optical trap. Simultaneously, Liphardt et al, ${ }^{10}$ used optical tweezers to pull the ends of a DNA-RNA hybrid chain, measuring the work required to unravel or unfold a specific domain in the chain. These experiments had complementary aims: The colloidal experiment was a classical model system constructed to cleanly demonstrate, as rigorously as possible in experiment, the Evans-Searles FT. In contrast Liphardt's RNA-unfolding experiment importantly demonstrated the potential application of Crooks FT to a complex biomolecular system, highlighting the potential practical use of FTs to a wider range of scientists. However, Liphardt et al constructed $\mathcal{O}(300)$ stretching profiles, an insufficient number to describe the distributions needed for Crooks FT, but fortunately sufficient to the show that the free energy change, $\Delta F$, obtained from Jarzynski's equality could be determined to within $k_{B} T$ from their $N$ experimental trajectories. In later work from the same group, Collin et al ${ }^{11}$ similarly used optical tweezers to construct experimental work distributions for the folding/unfolding of an RNA hairpin and an RNA three-helix junction. However, the $\Delta F$ for that transition was very large, $>60 k_{B} T$, and as such the FTs could not be used directly.

In this paper, we revisit the optical tweezers demonstration of the FT applied to a biopolymer system. We consider a simpler biopolymer system: a single ds-DNA molecule which is weakly stretched. This biopolymer does not display a first order or discontinuous transition as in the Liphardt and Collins systems, The stretching of the chain reduces the conformational states of the chain and consequently, there is an associated change in the chain's free energy that varies continuously with end-to-end distance of the chain. We demonstrate how the FT can be used to determine this free energy difference upon stretching of a single DNA chain.

\subsection{The Evans-Searles Fluctuation Theorem}

The Fluctuation Theorem (FT) of Evans \& Searles $^{3,4}$ describes how a finite sized system's irreversibility develops in time from a completely time-reversible system at short observation times, to an irreversible one at long times. It also shows how irreversibility emerges as the system size increases. That is, it bridges the microscopic and macroscopic descriptions, relating a system's time-reversible equations of motion to the Second Law, and provides a quantitative resolution to the long-standing irreversibility paradox. Specifically, the FT relates the relative probabilities, $p$, of observing trajectories of duration $t$ characterised by the dissipation function, $\Omega_{t}$, taking on arbitrary values $\mathcal{A}$ and $-\mathcal{A}$, respectively:

$$
\frac{p\left(\Omega_{t}=\mathcal{A}\right)}{p\left(\Omega_{t}=-\mathcal{A}\right)}=\exp (\mathcal{A}) .
$$

The dissipation function, $\Omega_{t}$, is, in general, a dimensionless dissipated energy, accumulated along the system's trajectory; expressions for $\Omega_{t}$ differ from system to system. However, in the case where there is no change of thermodynamic state of the system (or a purely dissipative process), $\Omega_{t}$ is simply the dissipative work, $\Omega_{t}=W_{\text {diss }}$. (A recent review ${ }^{8}$ provides a complete prescription of the relationship between $\Omega_{t}$ and work $W$.) Any trajectory of the system that is characterised by a particular value $\Omega_{t}=\mathcal{A}$ has, under time-reversible mechanics, a timereversed anti-trajectory with $\Omega_{t}=-\mathcal{A}$. In this way, the LHS of the FT has also been interpreted as a ratio of the probabilities of observing trajectories to their respective anti-trajectories. The dissipation function, $\Omega_{t}$, is 
an extensive property, i.e., its magnitude scales with system size, and it also scales with the observation time, $t$. Thus, eqn 1 also shows that as the system size gets larger or the observation time gets longer, anti-trajectories become rare and it becomes overwhelmingly likely that the system appears time-irreversible, in accord with the Second Law. That is, the evolution of a large macroscopic system proceeds preferentially in one direction.

Wang et al. ${ }^{9}$ first demonstrated eqn 1 in a simple OT experiment involving a single colloidal particle, weakly held in an optical trap that was translated uniformly with constant, but small, velocity $\mathbf{v}_{\text {opt }}$. Initially, the particle's position in the trap is distributed according to an equilibrium Boltzmann distribution with an average particle velocity of 0 . With trap translation, the particle is displaced from its equilibrium position until, at some time later, the average velocity of the particle is equal to the velocity of the optical trap. As dragging an isolated colloid particle with an optical trap is a purely dissipative process, $\Omega_{t}$ corresponds to the work done by the trap:

$$
\Omega_{t}=\frac{1}{k_{B} T} \int_{0}^{t} d s \mathbf{f}_{o p t} \cdot \mathbf{v}_{o p t},
$$

where the optical trapping force is $\mathbf{f}_{o p t}=-k \Delta \mathbf{x}, k$ being the trapping constant and $\Delta \mathbf{x}$ the measured displacement of the particle from the trap center. With the ability to resolve nanometer-scale particle displacements and femtoNewton scale optical forces, the dissipative work can be determined with sub- $k_{B} T$ resolution. Note that in the limiting case of vanishingly small velocity, $\mathbf{v}_{\text {opt }} \rightarrow 0$, and over long time scales where the particle position averages to the centre of the trap, $\Delta \mathbf{x}=0$, the dissipative work vanishes.

\subsection{Crooks Fluctuation theorem}

From classical thermodynamics, the work done by an external field to drive a system from one equilibrium state to another equilibrium state is equivalent to the change of free energy, $\Delta F$, between the states, only in the special case where the path is traversed quasi-statically. That is the path between the two states must be traversed so slowly that intermediate, as well as the initial and final states of the system, are all in thermodynamic equilibrium. However Crooks' Fluctuation Theorem (Crooks FT) $)^{5,6}$ describes the work required over non-equilbrium paths between states, i.e., paths that are traversed at arbitrary rate, ranging from quasi-static to "far-from-equilibrium" and which involve only a limited number of degrees of freedom. In essence, Crooks FT differs from Evans- Searles FT in that the work is not purely dissipative, but rather drives the system between states characterised by a free energy difference, $\Delta F$ :

$$
\frac{p_{f}(W=\mathcal{A})}{p_{r}(W=-\mathcal{A})}=\exp [(\mathcal{A}-\Delta F)]
$$

This expression is similar to Evans-Searles FT in that it relates distributions of trajectories, characterised by an energy, specifically the work, $W$. This work is formally defined as

$$
\dot{W}=\dot{\lambda} \frac{\partial \phi}{\partial \lambda}+\dot{W}_{d i s s}
$$

where $\lambda$ is an external control parameter, which, for example can be the end-to-end distance of the polymer. Crooks FT, eqn 3, relates trajectories initiated from two different equilibrium states, $A$ and $B$. That is, it considers (i) a distribution, $p_{f}$, of forward trajectories, $A \rightarrow B$, where the free energy change between equilibrium states $\mathrm{A}$ and $\mathrm{B}$ is $\Delta F=F_{B}-F_{A}$, and (ii) the distribution, $p_{r}$, of reverse trajectories, $B \rightarrow A$, where the respective equilibrium free energy change is $-\Delta F$. Like the FT, Crooks FT also quantifies how irreversibility evolves out of reversible equations of motion. A perfectly reversible (quasi-static) system is one where the work required to traverse $B \rightarrow A$ is equal but opposite in sign to the work required in the time-reversed trajectory, $A \rightarrow B$. Thus the RHS of eqn. 3 is unity for these reversible paths and $W=\Delta F$, in agreement with classical thermodynamics. For irreversible paths, that value of $W$ for which $p_{f}(W)=p_{r}(W)$ corresponds to $\Delta F$.

\subsection{Strategy for Analysis of DNA stretching using FTs}

Our strategy is to demonstrate the FTs by measuring the work done by the particle-filled optical trap upon translation. The dimensionless work is

$$
W=\frac{1}{k_{B} T} \int_{0}^{t} d s \mathbf{f}_{o p t} \cdot \mathbf{v}_{o p t} .
$$




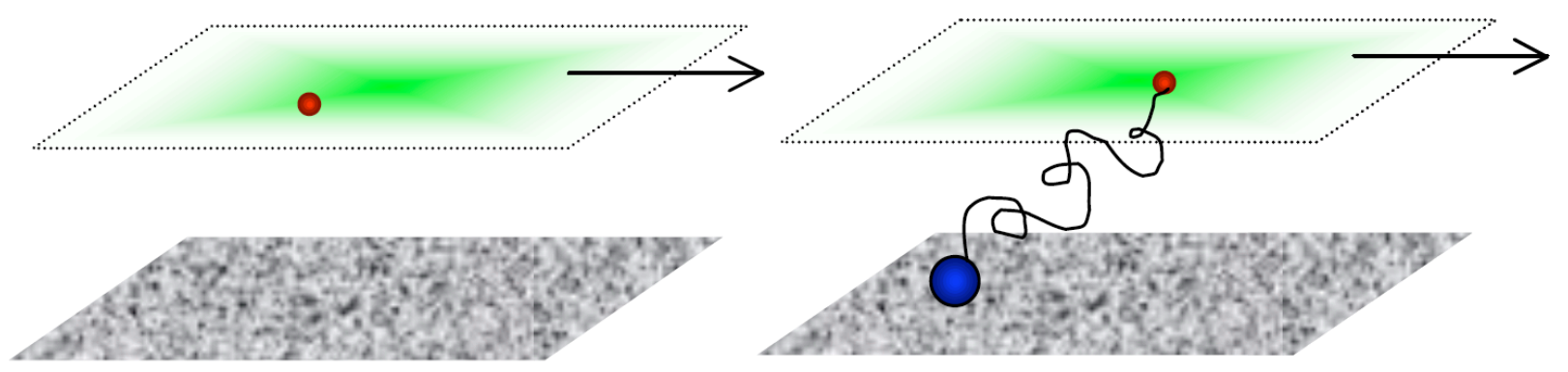

Figure 1. Schematic of Optical Tweezers experiment for (left) measuring the purely dissipative work in translating a particle-filled optical trap, where the particle is confined to a trapping plane which is displaced relative to its surroundings, and (right) measuring the work required to stretch a single $d s$-DNA molecule, end-tethered to two differently sized colloidal particles, where the large particle adheres irreversibly to the bottom plate of a fixed sample cell and the smaller, endtethered particle is trapped in the focal plane, which is displaced. The work required to stretch the chain consists of the work required to reduce the conformational states of the polymer as well as the dissipative work associated with the chain and optically trapped particle. Initially, when the chain is stretched, the work is predominantly dissipative, as in the case of the isolated particle in the translated trap.

If the optically-trapped particle is isolated, i.e. there is no chain tethered to the optically rapped particle as shown in the right of Figure 1, then the measured work is purely dissipative, $\dot{W}=\dot{W}_{\text {diss }}$ and, as shown by Wang et al., the distributions of measured $W$ follow the Evans-Searles FT. If however, the optically-trapped particle tethers one end of a fixed DNA molecule (as shown in the right of Figure 1), then the measurement consists of both dissipative work as well as work required to limit the conformational states of the DNA molecule upon stretching. In this case, the distributions of $W$ will not follow the Evans-Searles FT, but will instead follow Crooks FT. Referring to eqn 4, we make the representation that $\dot{\lambda}=v_{\text {opt }}$, that is the control parameter of the external agent is the translation of the optical trap relative to the stage, where $v_{\text {opt }} \sim \dot{L}$ is roughly the rate of change of the chain's end-to end distance. As $\phi(L)$ is the potential function of the chain depends upon its end-to-end distance,

$$
W=\int_{0}^{t} d s \dot{L} \frac{\partial \phi}{\partial L}+\int_{0}^{t} d s \dot{W}_{d i s s}
$$

In principle, for very small $\dot{L} \sim v_{\text {opt }}$ and long time, $t$, (i.e., very slow chain stretching) the dissipative components of the chain and optically-trapped bead will vanish and the measured $W$ would match the quasi-static equilibrium result, $W=\Delta F$. In practice, when the imposed change in the end-to-end distance of the chain is negligible, $W \sim W_{\text {diss }}$ and the experiment differs little from the Wang experiment where a single isolated bead is fluctuating in a translating optical trap. Under those conditions, the distribution of experimentally measured $W$ should fit the Evans-Searles FT. However, when the chain's end-to-end distance changes appreciably, then both terms on the RHS of eqn 6 are important, and the distribution of measured $W$ should fit the Crooks FT, rather than the Evans-Searles FT.

\section{EXPERIMENT}

\section{Equipment and Samples}

The Optical Tweezers apparatus is similar to that described in Wang et al..$^{9}$ It consists of a Nikon DAPHOT 300 inverted microscope equipped with a $100 \times(\mathrm{NA}=0.75)$ oil immersion objective lens and a $1 \mathrm{~W}$ infrared laser $(\lambda=980 \mathrm{~nm})$ isolated on a air-cushioned optical table. A servo-motor controls the positioning of the microscope stage with fine translation achieved by feeding a voltage signal from an arbitrary function generator to the stagemounted piezocrystals. A quadrant photodiode sensor detects particle displacement from the trap centre, $\Delta x$, with resolution $15 \mathrm{~nm}$ at a $2 \mathrm{kHz}$ sampling rate. The high numerical aperture objective strongly traps the latex 
particle in the focal plane, while weakly trapping in the $x=y$ plane. The optical trapping constant in the $x-y$ plane is determined by monitoring the motion of a free particle's position and applying equipartition, as well as by analysis of the power spectral density.

The ends of double stranded $\lambda$-DNA were tethered to two different types of trap-able latex colloidal beads, a large $10 \mu \mathrm{m}$-diameter bead coated with avidin (CP01N Bangs Lab Inc., Illinois USA) which binds to a biotinylated end of $d s$-DNA, and a smaller $5.5 \mu \mathrm{m}$-diameter carboxylate modified latex (CML) particle, coated with antidigoxigenin according to the protocol from Interfacial Dynamic Inc (USA). These smaller coated beads bind to the other end of the $d s$-DNA molecule, modified with the insertion of digoxigenin. The end-modified $\lambda$-DNA was kindly provided by Prof. Antoine Van Oijins group Harvard Medical School and a diluted solution was made by adding $15 \mu \mathrm{l}$ of TE buffer solution to $5 \mathrm{ul}$ of the provided DNA stock. The bead-DNA-bead was constructed by pipetting $4 \mu \mathrm{l}$ of diluted DNA stock, $5 \mu \mathrm{l}$ of $5.5 \mu \mathrm{m}$-dia bead solution (anti-digoxigenin coated) and $10 \mu \mathrm{l}$ of 10 $\mu \mathrm{m}$ bead solution (avidin coated) into a vial which was placed on a shaker (100 rpm) at ambient temperature for gentle shaking for 2 hours to increase the mobilities of both DNA and beads. The DNA and bead mixture was further diluted by adding $81 \mu \mathrm{l}$ of $\mathrm{TE}$ buffer solution and $100 \mu \mathrm{l}$ of $200 \mathrm{mM} \mathrm{NaCl}$ solution. The $\mathrm{NaCl}$ solution was added to facilitate the sticking of the larger, $10 \mu \mathrm{m}$ avidin-coated beads to the bottom of the sample cell..

The sample cell in which the DNA molecules is stretched was constructed by sandwiching double-sided adhesive sheet of $120 \mu \mathrm{m}$ thickness (Grance Bio-Lab, USA) with a $50 \times 3 \mathrm{~mm}^{2}$ channel cut-out, between a thin microscope slide $(60 \times 24 \times 0.15 \mathrm{~mm})$ and a normal microscope slide. The thin slide served as the lower bottom of our sample cell; the thinness of this slide enabled us to trap with our objective's working distance. 4 holes in the top slide allow us to inject /withdraw solution containing bead-tethered DNA. The sample solution was drawn to the sample cell via a syringe pump at a rate $\sim 1 \mu \mathrm{l} / \mathrm{s}$ until the flow channel was fully filled. After an hour of incubation time, we could observe that (a) most of the large $10 \mu \mathrm{m}$ beads stuck to the substrate; (b) the smaller $5.5 \mu \mathrm{m}$ beads were freely diffusing; and (c) that there were pairs of large-small bead pairs, with the smaller bead fluctuating about the larger bead. These were identified as beads fixed to DNA for potential stretching.

We confirmed bead-DNA-bead structures by first trapping the smaller $5.5 \mu \mathrm{m}$ bead with an optical trap of modest strength ( $k=10$ to $15 \mathrm{pN} / \mu \mathrm{m}$ ) and translating the stage slowly (usually less than $1 \mu \mathrm{m} / \mathrm{s}$ ) so that larger, surface-bound bead translates away from the smaller, optically trapped bead. If the optically trapped bead pops out of the trap with moderate stage translation and rebounds back to the surface-fixed bead, then we have confirmed a stable bead-DNA-bead structure for suitable for repetive stretching.

Repetitive stretching of this single bead-DNA-bead assembly proceeds by translating stage under specified conditions, while at the same time, recording the beads displacement from the trap center at $500 \mathrm{~Hz}$. As we are interested only in weak stretching, the small bead is trapped with a relatively weak trap, $k \sim 0.8 \mathrm{pN} / \mu \mathrm{m}$, while the stage is translated forward at a speed of $0.625 \mathrm{um} / \mathrm{s}$ for up to $0.5 \mathrm{~s}$, held-stationary for $2 \mathrm{~s}$ to allow the chain to equilibrate, and then translated in the reverse direction, at the same speed. This resulted in a maximum stage displacement (indicative of the maximum end-to-end distance of the DNA chain) of $\sim 312 \mathrm{~nm}$. This stretch and release cycle were repeated 3700 times for a single DNA chain. The minimum distance between particle surfaces observed to be $\sim 2.7 \mu \mathrm{m}$ and the maximum distance a little over $3 \mu \mathrm{m}$.

\subsection{Experimental results and analysis}

The accumulated work over any arbitrary time, $t$, up to $0.5 \mathrm{~s}$, is calculated for a stretch or release trajectory using

$$
W=\frac{1}{k_{B} T} \int_{0}^{t} d s \mathbf{f}_{\text {opt }} \cdot \mathbf{v}_{\text {opt }}=-\frac{k_{\text {opt }} v_{\text {opt }}}{k_{B} T} \int_{0}^{t} d s \Delta x(s),
$$

where the system (chain and optically trapped particle) must be at equilibrium at $t=0$. Histograms of $W$ are compiled for stretch and release trajectories and from these histograms, the FTs are demonstrated. For brevity, we consider here two types of trajectories: short trajectories where the work of stretch and release is accumulated over trajectories of duration $t=100 \mathrm{~ms}$, and long trajectories where $t=500 \mathrm{~ms}$. Figure 2 shows the histograms associated for 3700 trajectories where the chain is stretched $\sim 0.3 \mu \mathrm{m}$ over $1 / 2$ second (black points) and released over the same distance and time (red points). Notice that the width of the distributions is appreciable, several $k_{B} T$; that the most probable value of $W$ for stretch is larger than that of release; and that the release trajectories 


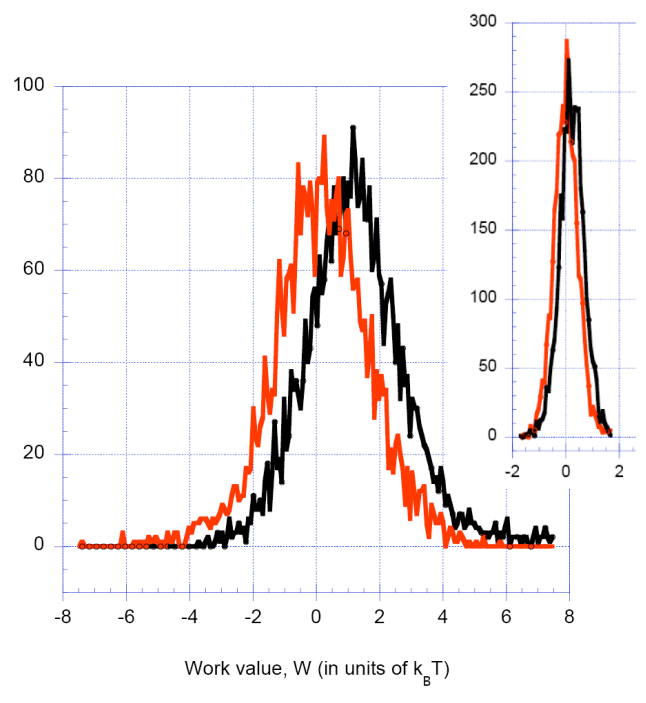

Figure 2. Distribution of measured $W$, for 3700 repetitive stretch (black) and release (red/gray) trajectories of a single $d s-$ DNA molecule. Each trajectory is initiated at equilibrium, with stage translation $v_{\text {opt }}=0.625 \mu \mathrm{m} / \mathrm{s}$ for $t=500 \mathrm{~ms}$. Inset shows distributions for 3700 repetitive stretch and release trajectories with $v_{\text {opt }}=0.625 \mu \mathrm{m} / \mathrm{s}$ but much shorter $t=100 \mathrm{~ms}$ trajectories. Notice that there is a clear distinction between the distributions of stretched and released trajectories for more strongly stretched chain, $t=500 \mathrm{~ms}$, whilst the distirbutions for stretch and release do not differ significantly for $t=100 \mathrm{~ms}$

are more likely to have negative values of $W$, as one would expect. By Crooks' FT, the value of $W$ at which the stretch and release distributions are equivalent corresponds to the change in free energy, $\Delta F$, for extending the length of the chain by $\sim 0.3 \mu \mathrm{m}$. Inspection of the histrogram, Figure 2 , shows $\Delta F$ to be a less than $k_{B} T$. The inset shows the distributions of $W$ for a much shorter time of stretch and release, where the chain is elongated $\sim 0.06 \mu \mathrm{m}$ over $t=100 \mathrm{~ms}$. The distribution of $W$ for such short stretch and release trajectories are roughly identical with most probable values of $W$ being roughly $0 k_{B} T$.

These histograms can be used to demonstrate the FTs as shown in Figure 3 for $t=100 \mathrm{~ms}$ (left) and $t=500$ ms (right). The LHS of Crooks FT is constructed from the ratio of the number of stretch trajectories of work value $W \pm d W, N_{\text {stretch }}(W)$, to the number of release trajectories of work value $-W \mp d W, N_{\text {release }}(-W)$; the natural $\log$ of this ratio plotted against $W$ is predicted by the FTs to be a line of slope 1.0. Note that of the 3700 trajectories, values of large $W$, as for example $W=\mathcal{O}\left(5 k_{B} T\right)$ in the $t=500 \mathrm{~ms}$ data, is not sampled: that is $N_{\text {stretch }}\left(W=5 k_{B} T\right)=0$ indicating "null" sampling. Moreover, the data associated with large values of $|W|$ for which $N(W)>0$ is usually displaced from the predicted line. These "fringe" points are interspersed amongst "null" samples and represent values of $W$ that are rarely sampled. Consequently, these fringe points usually have a value of $\left(N_{\text {stretch }}(W) / N_{\text {release }}(-W)\right.$ that is offset from the FT prediction. Increasing the sample size, or number of trajectories sampled, would reduce the statistical error of these fringe points, bringing them closer to the FT prediction, but may also populate a point that was null in the smaller sample size. Thus, the FTs are best statistically represented by sampled trajectories with small values of $|W|$.

In Figure 3, we have included the predictions of the FTs (red/gray line) for comparison. Note that for the short, $t=100 \mathrm{~ms}$ trajectories, where there is very small elongation $\Delta L=0.06 \mu \mathrm{m}$ of the $L=16 \mu \mathrm{m}$ chain, the FT prediction passes through $\ln N_{\text {stretch }}(W) / N_{\text {release }}(-W)=0$ at $W=0$. That is, by Crooks FT, $\Delta F=0$, or there is no measureable thermodynamic change achieved in the process of a stretch (or release) trajectory. Under those conditions, the stretch and release trajectories are not distinguishable and are identical to trajectories generated with a simple translation of a particle-filled trap, in the absence of the tethered chain. Indeed, in the case where the forward/reverse or stretch/release trajectories are indistinguishable, we can combine the stretch and release distributions, $P_{\text {stretch }}(W)+P_{\text {release }}(W)=P(W)$ to generate a more populated distribution which can be fit to Evans-Searles FT. In contrast, the longer stretch/release trajectories compare favorable with an FT prediction 

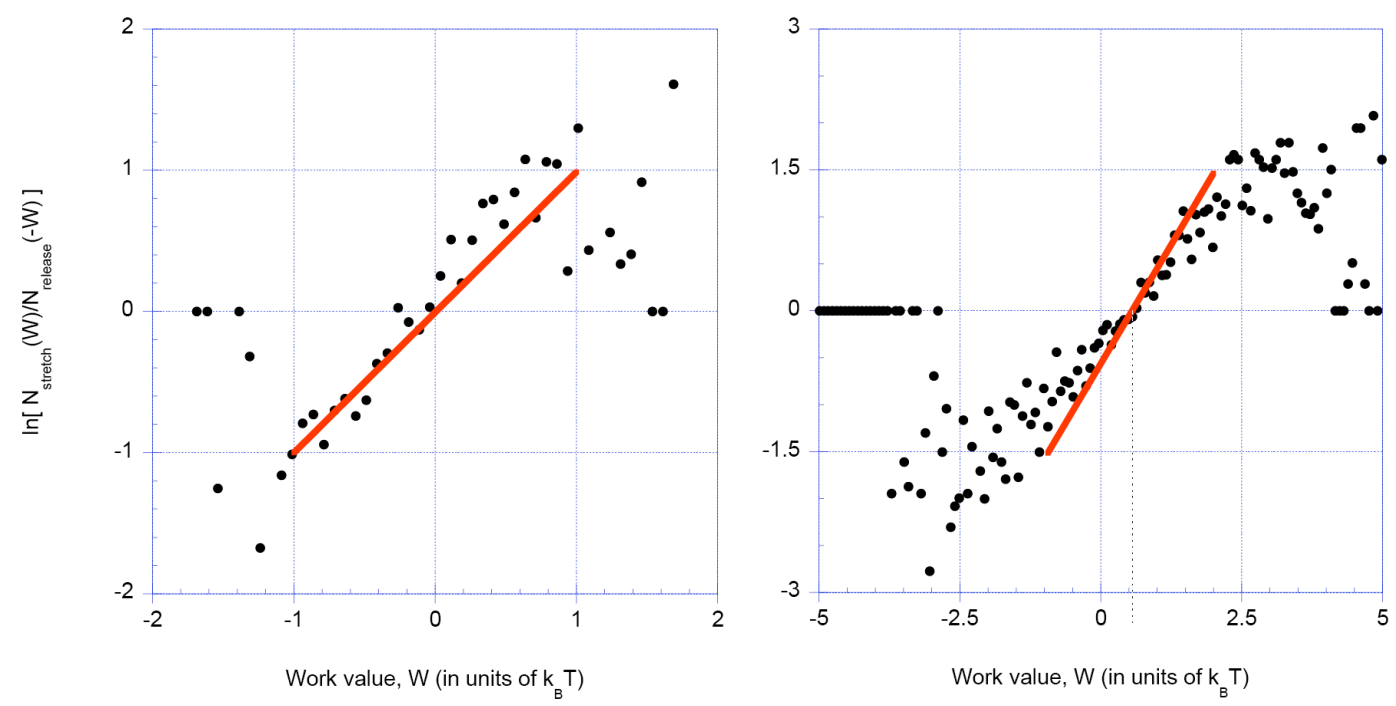

Figure 3. LHS of the FTs, $\ln N_{\text {stretch }}(W) / N_{\text {release }}(-W)=0$ versus $W$, measured work for $t=100 \mathrm{~ms}$ (left) and $t=500$ $\mathrm{ms}$ (right). For $t=100 \mathrm{~ms}$, the experimental data (black dots) is in agreement with the predictions of the Evans-Searles FT, as well as Crooks FT with $\Delta F=0$ (red line). For $t=500 \mathrm{~ms}$, the experimental data is in agreement with the Crooks FT with $\Delta F \sim 0.5 k_{B} T$.

that passes through $\ln N_{\text {stretch }}(W) / N_{\text {release }}(-W)=0$ at $W=0.5 k_{B} T$. That is, by Crooks FT, $\Delta F=0.5 k_{B} T$. Indeed, this exercise suggest an experimental method for the measurement of the end-to-end distance dependent free energy, $\Delta F(L)$, from which a force law can be constructed, $f(L)=-\partial \Delta F(L) / \partial L$. This might prove a useful method for constructing force laws governing complex biopolymer chains.

\subsection{Acknowledgments}

The authors thank Prof. Nick Dixon of the University of Wollongong for several discussions and Prof. Antoine Van Oijin and his group at the Harvard Medical School (HMS) for providing end-modified $\lambda$ DNA, as well as Dr. Samir Hamdan (HMS) for his detailed assistance. The authors also thank Prof. Denis J. Evans for valuable discussions and collaborations over the past few years. The authors gratefully acknowledge financial support from the Australian Research Council.

\section{REFERENCES}

[1] de Groot, S. R. and Mazur, P., [Non-equilibrium Thermodynamics], Dover, New York (1984).

[2] Kondepudi, D. and Prigogine, I., [Modern Thermodynamics], Wiley, New York (1998).

[3] Evans, D. J. and Searles, D. J., "Equilibrium microstates which generate 2nd law violating steady-states," Phys. Rev. E 50, 1645-1648 (Aug. 1994).

[4] Evans, D. J. and Searles, D. J., "The fluctuation theorem," Adv. Phys. 51, 1529-1585 (Nov. 2002).

[5] Crooks, G. E., "Nonequilibrium measurements of free energy differences for microscopically reversible Markovian systems," J. Stat. Phys. 90, 1481-1487 (Mar. 1998).

[6] Crooks, G. E., "Entropy production fluctuation theorem and the nonequilibrium work relation for free energy differences," Phys. Rev. E 60, 2721-2726 (Sept. 1999).

[7] Jarzynski, C., "Nonequilibrium equality for free energy differences," Phys. Rev. Lett. 78, 2690-2693 (Apr. 1997).

[8] Sevick, E. M., Prabhakar, R., Williams, S. R., and Searles, D. J., "Fluctuation theorems," Annual Review of Physical Chemistry 59, 603-633 (2008).

[9] Wang, G. M., Sevick, E. M., Mittag, E., Searles, D. J., and Evans, D. J., "Experimental demonstration of violations of the second law of thermodynamics for small systems and short time scales," Phys. Rev. Lett. 89, 050601 (July 2002). 
[10] Liphardt, J., Dumont, S., Smith, S. B., Tinoco, I., and Bustamante, C., "Equilibrium information from nonequilibrium measurements in an experimental test of Jarzynski's equality," Science 296, 1832-1835 (June 2002).

[11] Collin, D., Ritort, F., Jarzynski, C., Smith, S. B., Tinoco, I., and Bustamante, C., "Verification of the Crooks fluctuation theorem and recovery of RNA folding free energies," Nature 437, 231-234 (Sept. 2005). 